\title{
An Explicit Time Domain Finite Element Boundary Integral Method with Element Level Domain Decomposition for Electromagnetic Scattering Analysis
}

\author{
Ming Dong*, Ping $\mathrm{Li}^{\dagger}$, Hakan Bagci* \\ * Division of Computer, Electrical, and Mathematical Science and Engineering, King Abdullah University of Science and \\ Technology, Thuwal, Saudi Arabia, \{ming.dong, hakan.bagci\}@kaust.edu.sa \\ $\dagger$ Key Laboratory of Ministry of Education of Design and Electromagnetic Compatibility of High-Speed Electronic Systems, \\ Shanghai Jiao Tong University, Shanghai, China, ping.li@sjtu.edu.cn
}

\begin{abstract}
A numerical scheme, which hybridizes the element level dual field time domain finite element domain decomposition method (ELDFDD/TDFEM) and time domain boundary integral (TDBI) method to accurately and efficiently analyze open-region transient electromagnetic scattering problems, is proposed. The element level decomposition permits an efficient leapfrog-like explicit marching scheme to be used to integrate Maxwell equations in time. The hybridization with TDBI method ensures that an accurate solution can be obtained in the smallest computation domain possible. The accuracy and applicability of the proposed hybrid method is demonstrated by numerical experiments.
\end{abstract}

Index Terms-domain decomposition, time-domain finiteelement method, boundary integral.

\section{INTRODUCTION}

Numerical schemes that are capable of analyzing transient electromagnetic scattering from an inhomogeneous object residing in an unbounded background medium are indispensable in many branches of electromagnetics, optics, and photonics. Time domain finite element method (TDFEM) is often used for this purpose [1], [2], [3]. However, when TDFEM is used to analyze an open-region scattering problem, the unbounded background medium must be truncated into a finite computation domain. This is often done by "wrapping" the computation domain with absorbing boundary conditions (ABCs) or perfectly matched layers (PMLs) [4], [5], [6]. The accuracy of the $A B C$ decreases significantly for waves obliquely incident on the boundaries [4] while the PML might introduce late time instabilities in the solution and its accuracy deteriorates at low frequencies[5], [6], [7]. Another approach for truncating an unbounded background medium is to "hybridize" the TDFEM with the time domain boundary integral (TDBI) method [8], [9]. This hybrid approach is mathematically exact and the truncated boundary can be located very close to the scatterer surface (regardless of its shape) [9].

In this work, the element level dual field time domain finite element domain decomposition method (ELDFDD/TDFEM) [10] is hybridized with the TDBI method for the first time to accurately and efficiently analyze open-region transient scattering problems. The computation domain is divided into tetrahedral elements and in each element the dual-field secondorder vector wave equation is enforced/solved. Electric and magnetic fields are updated at staggered time steps so that the communication between elements can be realized by introducing equivalent surface currents on the interfaces. An efficient leapfrog-like explicit time marching scheme is constructed since each element is related to its neighboring element in an explicit manner [11], [10]. Additionally, the fields on the computation domain boundary are computed using the TDBI method from the equivalent currents introduced on a Huygens surface enclosed inside the computation domain. This ensures that the radiation condition is exactly enforced (mathematically speaking — it is still subject to discretization error). Numerical results demonstrate the accuracy and applicability of the proposed method in various scattering scenarios.

\section{FORMULATION}

A 3D scatterer with permittivity $\varepsilon(\mathbf{r})$ and permeability $\mu(\mathbf{r})$ is residing in an unbounded background medium. Let $V$ denote the computation domain truncated by a fictitious surface $S$ enclosing the scatterer. $\partial \Gamma$ denotes the Huygens surface that is enclosed inside $S$ and fully encloses the scatterer. The whole computation domain $V$ is divided into $M$ nonoverlapping tetrahedrons. Let $\mathbf{E}_{m}(\mathbf{r}, t)$ and $\mathbf{H}_{m}(\mathbf{r}, t)$ denote electric and magnetic fields in the $m$ th element with volume $V_{m}, m=1,2, \ldots M$. Four faces of the $m$ th element are denoted by $S_{m f}, f=1,2,3,4$. Note that in the rest of text space and time are dropped for the sake of simplicity. In element $m$, the local field $\mathbf{E}_{m}$ satisfies source-free second-order vector wave equation

$$
\nabla \times\left(\frac{1}{\mu_{m}} \nabla \times \mathbf{E}_{m}\right)+\varepsilon_{m} \partial_{t}^{2} \mathbf{E}_{m}=0
$$

where $\varepsilon_{m}$ and $\mu_{m}$ are permittivity and permeability in $V_{m}$, respectively. Applying Galerkin testing to (1) with vector basis 
function $\mathbf{N}_{i}$ yields

$$
\begin{gathered}
\iiint_{V_{m}} \frac{1}{\mu_{m}}\left(\nabla \times \mathbf{N}_{i}\right) \cdot\left(\nabla \times \mathbf{E}_{m}\right)+\varepsilon_{m} \mathbf{N}_{i} \cdot \partial_{t}^{2} \mathbf{E}_{m} d V \\
=\sum_{f=1}^{4} \iint_{S_{m f}} \mathbf{N}_{i} \cdot \hat{n}_{m f} \times \partial_{t} \mathbf{H}_{m}^{+} d S
\end{gathered}
$$

where $\hat{n}_{m f}$ is the outward unit normal vector on facet $S_{m f}$ and $\mathbf{H}_{m}^{+}$denotes the magnetic field in the neighboring element which is connected to the $m$ th element through face $S_{m f}$. Note that to derive (2), we make use of Maxwell equation

$$
\frac{1}{\mu_{m}} \nabla \times \mathbf{E}_{m}=-\partial_{t} \mathbf{H}_{m}^{+}
$$

on $S_{m f}$ to impose the tangential continuity of the fields on the interface. Here, we need to mention that if the face $S_{m f}$ is located on the computation domain boundary, the exterior fields need to be computed using the TDBI method to enforce the exact radiation condition. Incident field $\left\{\mathbf{E}^{\text {inc }}, \mathbf{H}^{\text {inc }}\right\}$ is also introduced through tetrahedron faces residing on the computation domain boundary.

To compute the fields on $S$ using the TDBI method, a set of equivalent electric and magnetic currents $\mathbf{J}^{\mathrm{e}}$ and $\mathbf{M}^{\mathrm{e}}$ is introduced on the Huygens surface $\partial \Gamma$. These currents are calculated from the local fields solved/updated by the TDFEM as

$$
\begin{aligned}
\mathbf{J}^{\mathrm{e}} & =\sum_{l=1}^{K} \hat{n}_{l} \times \mathbf{H}_{l} \\
\mathbf{M}^{\mathrm{e}} & =-\sum_{l=1}^{K} \hat{n}_{l} \times \mathbf{E}_{l}
\end{aligned}
$$

where $K$ denotes the total number of tetrahedral elements residing on $\partial \Gamma$ and $\left\{\mathbf{E}_{l}, \mathbf{H}_{l}\right\}$ denote the fields on the $l$ th one of those elements. Then the boundary fields $\left\{\mathbf{E}^{s}, \mathbf{H}^{s}\right\}$ are constructed from $\mathbf{J}^{\mathrm{e}}$ and $\mathbf{M}^{\mathrm{e}}$ using

$$
\begin{aligned}
\mathbf{E}^{\mathrm{s}} & =\mathcal{L}\left(\mathbf{J}^{\mathrm{e}}\right)-\mathcal{K}\left(\mathbf{M}^{\mathrm{e}}\right) \\
\mathbf{H}^{\mathrm{s}} & =\mathcal{L}\left(\mathbf{M}^{\mathrm{e}}\right) / \eta_{0}^{2}+\mathcal{K}\left(\mathbf{J}^{\mathrm{e}}\right)
\end{aligned}
$$

where $\mathcal{L}$ and $\mathcal{K}$ operators are defined as

$$
\begin{aligned}
\mathcal{L}(\mathbf{X}) & =-\frac{\mu_{0}}{4 \pi} \int_{\partial \Gamma} \frac{\partial_{t} \mathbf{X}\left(\mathbf{r}^{\prime}, t-R / c_{0}\right)}{R} d \mathbf{r}^{\prime} \\
& +\frac{\nabla}{4 \pi \varepsilon_{0}} \int_{\partial \Gamma} d \mathbf{r}^{\prime} \int_{0}^{t-R / c_{0}} \frac{\nabla^{\prime} \cdot \mathbf{X}\left(\mathbf{r}^{\prime}, t^{\prime}\right)}{R} d t^{\prime} \\
\mathcal{K}(\mathbf{X}) & =\nabla \times \frac{1}{4 \pi} \int_{\partial \Gamma} \frac{\mathbf{X}\left(\mathbf{r}^{\prime}, t-R / c_{0}\right)}{R} d \mathbf{r}^{\prime} .
\end{aligned}
$$

Here $\eta_{0}=\sqrt{\mu_{0} / \varepsilon_{0}}$ and $c_{0}=1 / \sqrt{\varepsilon_{0} \mu_{0}}$ denote the wave impedance and wave speed in the background medium, respectively. $R=\left|\mathbf{r}-\mathbf{r}^{\prime}\right|$ is the distance between field point $\mathbf{r}$ and source point $\mathbf{r}^{\prime}$.
Following the same procedure, the dual form of (2) for Hfield can be written as

$$
\begin{gathered}
\iiint_{V_{m}} \frac{1}{\varepsilon_{m}}\left(\nabla \times \mathbf{N}_{i}\right) \cdot\left(\nabla \times \mathbf{H}_{m}\right)+\mu_{m} \mathbf{N}_{i} \cdot \partial_{t}^{2} \mathbf{H}_{m} d V \\
=-\sum_{f=1}^{4} \iint_{S_{m f}} \mathbf{N}_{i} \cdot \hat{n}_{m f} \times \partial_{t} \mathbf{E}_{m}^{+} d S
\end{gathered}
$$

After space discretization, we can cast (2) and (7) into the following matrix form

$$
\begin{aligned}
{\left[S_{m}^{e}\right]\left\{e_{m}\right\}+\left[M_{m}^{e}\right] \partial_{t}^{2}\left\{e_{m}\right\} } & =\partial_{t}\left\{f_{m}^{e}\right\} \\
{\left[S_{m}^{h}\right]\left\{h_{m}\right\}+\left[M_{m}^{h}\right] \partial_{t}^{2}\left\{h_{m}\right\} } & =\partial_{t}\left\{f_{m}^{h}\right\}
\end{aligned}
$$

where $\left\{e_{m}\right\}$ and $\left\{h_{m}\right\}$ are the time dependent unknown vectors in the $m$ th element. $\left[S_{m}^{e}\right],\left[S_{m}^{h}\right],\left[M_{m}^{e}\right],\left[M_{m}^{h}\right]$ denote the stiffness and mass matrices for electric and magnetic fields in $V_{m}$, respectively. $\left\{f_{m}^{e}\right\}$ and $\left\{f_{m}^{h}\right\}$ result from the right-hand side surface integral of (2) and (7).

In time domain, we apply central difference method to discretize the system. Electric field is "sampled" at integer time indices while magnetic field is "sampled" at half-integer indices, resulting in the final leapfrog like time marching scheme

$$
\begin{aligned}
{\left[\mathbf{M}_{m}^{e}\right]\left\{e_{m}\right\}^{n+1}=\left(2\left[\mathbf{M}_{m}^{e}\right]-\Delta t^{2}\left[\mathbf{S}_{m}^{e}\right]\right)\left\{e_{m}\right\}^{n} } \\
-\left[\mathbf{M}_{m}^{e}\right]\left\{e_{m}\right\}^{n-1}+\Delta t\left(\left\{f_{m}^{e}\right\}^{n+\frac{1}{2}}-\left\{f_{m}^{e}\right\}^{n-\frac{1}{2}}\right) \\
{\left[\mathbf{M}_{m}^{h}\right]\left\{h_{m}\right\}^{n+\frac{3}{2}}=\left(2\left[\mathbf{M}_{m}^{h}\right]-\Delta t^{2}\left[\mathbf{S}_{m}^{h}\right]\right)\left\{h_{m}\right\}^{n+\frac{1}{2}} } \\
-\left[\mathbf{M}_{m}^{h}\right]\left\{h_{m}\right\}^{n-\frac{1}{2}}+\Delta t\left(\left\{f_{m}^{h}\right\}^{n+1}-\left\{f_{m}^{h}\right\}^{n}\right) .
\end{aligned}
$$

Equation (9) shows that the local electric and magnetic fields are updated at staggered time steps. For a given time step $n$, all the field vectors required on the right-hand side are already known, same for the given time step $n+1 / 2$. Thus, an explicit time-marching scheme is constructed.

\section{NUMERICAL RESULTS}

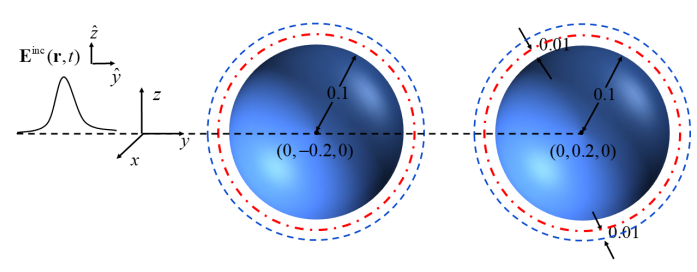

Fig. 1. Two separated dielectric spheres. (Unit: m)

This section presents a numerical example to demonstrate the accuracy and applicability of the proposed method. The excitation is a baseband Gaussian pulse plane wave whose electric field is expressed as $\mathbf{E}^{\text {inc }}=\hat{z} E_{0} G\left(t-\hat{y} \cdot \mathbf{r} / c_{0}\right)$, where $\hat{z}$ and $\hat{y}$ denote the direction of propagation and polarization, $E_{0}=1 \mathrm{~V} / \mathrm{m}$ is the amplitude, $G(t)=\exp \left[-\left(t-t_{0}\right)^{2} / \sigma^{2}\right]$ is the Gaussian pulse with delay $t_{0}=2.55 \mathrm{~ns}$ and duration $\sigma=$ $0.51 \mathrm{~ns}$. The effective bandwidth of thus pulse is $1.25 \mathrm{GHz}$. The computation domain is discretized into 56828 tetrahedrons and 
zeroth-order vector edge basis functions are used to discretize the fields.

Fig. 1 illustrates two (separated) dielectric spheres with $\varepsilon_{r}=2.0$ and $\mu_{r}=1.0$, which reside in free space. The TDBI method allows the computation domain to be defined as a union of two disconnected equally sized spheres, each of which contains one of the dielectric spheres. This significantly reduces the size of the computation domain. Also note that both the Huygens surfaces and computation domain boundaries conformal to the shapes of sphere are placed $0.01 \mathrm{~m}$ and $0.02 \mathrm{~m}$ away from the sphere surfaces, respectively. After the transient simulation is completed, we can calculate the bi-static radar cross section (RCS) at $500 \mathrm{MHz}$ and $1 \mathrm{GHz}$ on both $\mathrm{E}$ and $\mathrm{H}$ planes using the Fourier transformed transient currents recorded on the Huygens surfaces.

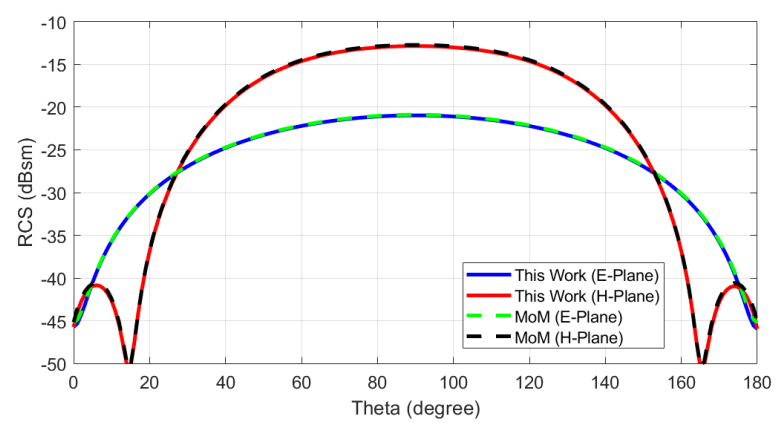

(a) RCS at $500 \mathrm{MHz}$

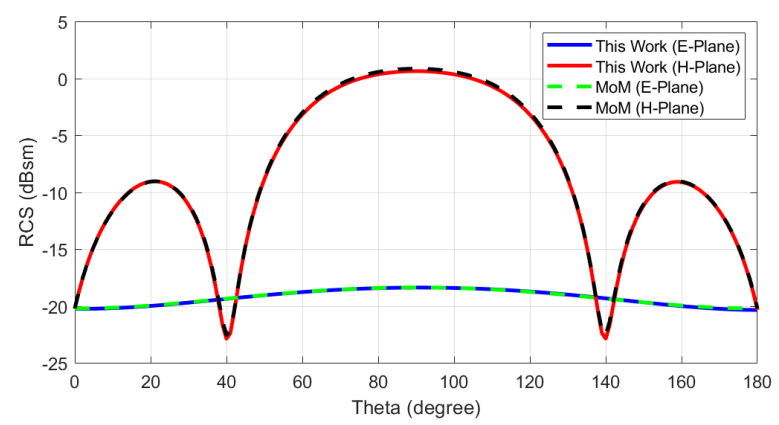

(b) $\mathrm{RCS}$ at $1 \mathrm{GHz}$

Fig. 2. RCS computed on E and H-planes by the proposed method and a frequency domain integral equation solver at (a) $500 \mathrm{MHz}$ and (b) $1 \mathrm{GHz}$.
Fig. 2 shows the RCS results obtained with proposed method agree very well with those obtained from a frequency domain MoM solver. It clearly verifies the accuracy of the proposed method.

\section{CONCLUSION}

In this paper, we present a novel scheme which combines the advantages of both dual-field element-level domaindecomposition TDFEM and TDBI method to accurately and efficiently analyze electromagnetic scattering problems. Numerical results demonstrating the accuracy and applicability of the proposed method are presented.

\section{REFERENCES}

[1] J.-M. Jin, The Finite Element Method in Electromagnetics. New York, United States: John Wiley \& Sons Inc, 2014.

[2] Jin-Fa Lee, R. Lee, and A. Cangellaris, "Time-domain finite-element methods," IEEE Trans. Antennas Propag., vol. 45, no. 3, pp. 430-442, Mar. 1997.

[3] L. E. R. Petersson and Jian-Ming Jin, "A three-dimensional time-domain finite-element formulation for periodic structures," Microw. Opt. Tech. Lett., vol. 54, no. 1, pp. 12-19, Jan. 2006.

[4] R. L. Higdon, "Absorbing boundary conditions for difference approximations to the multidimensional wave equation," Math. Comput., vol. 47, pp. 437-459, 1986.

[5] S. Abarbanel, D. I. Gottlieb, and J. S. Hesthaven, "Long time behavior of the perfectly matched layer equations in computational electromagnetics," J. Sci. Comput., vol. 17, pp. 405-422, 2002.

[6] S. D. Gedney and B. Zhao, "An auxiliary differential equation formulation for the complex-frequency shifted PML," IEEE Trans. Antennas Propag., vol. 58, no. 3, pp. 838-847, Mar. 2010.

[7] K. Sirenko, M. Liu, and H. Bagci, "Incorporation of exact boundary conditions into a discontinuous Galerkin finite element method for accurately solving $2 \mathrm{~d}$ time-dependent maxwell equations," IEEE Trans. Antennas Propag., vol. 61, no. 1, pp. 472-477, Jan. 2013.

[8] D. Jiao, A. A. Ergin, B. Shanker, E. Michielssen, and Jian-Ming Jin, "A fast higher-order time-domain finite element-boundary integral method for 3-D electromagnetic scattering analysis," IEEE Trans. Antennas Propag., vol. 50, no. 9, pp. 1192-1202, Sep. 2002.

[9] P. Li, Y. Shi, L. J. Jiang, and H. Bagci, "A hybrid time-domain discontinuous Galerkin-boundary integral method for electromagnetic scattering analysis," IEEE Trans. Antennas Propag., vol. 62, no. 5, pp. 2841-2846, May 2014.

[10] Z. Lou and J.-M. Jin, "A new explicit time-domain finite-element method based on element-level decomposition," IEEE Trans. Antennas Propag., vol. 54, no. 10, pp. 2990-2999, Oct. 2006.

[11] Z. Lou and J.-M. Jin, "A novel dual-field time-domain finite-element domain-decomposition method for computational electromagnetics," IEEE Trans. Antennas Propag., vol. 54, no. 6, pp. 1850-1862, June 2006. 